\title{
Análisis de la susceptibilidad al daño por humedad de una mezcla asfáltica a partir del ensayo MIST y del programa iPAS 2D ${ }^{\circledR}$
}

Moisture damage analysis for an asphalt mixture through the MIST test and the iPAS $2 D^{\circledR}$ software

\section{Ana Sofía Figueroa Infante}

Universidad de la Salle, Bogotá, Colombia

afigueroa@unisalle.edu.co
Fredy Alberto Reyes Lizcano

Universidad Javeriana, Bogotá, Colombia

Fredy.reyes@javeriana.edu.co

Fecha de recepción: 22 de enero de 2016 / Fecha de aprobación: 14 de marzo de 2016

\section{RESUMEN}

El daño por humedad es uno de los problemas más comunes en los pavimentos asfálticos en países tropicales y en zonas expuestas a periodos prolongados de lluvia. Esta investigación presenta los resultados de la susceptibilidad a la humedad de una mezcla asfáltica para rodadura, diseñada por la metodología Superpave ${ }^{\bullet}$ y fabricada con ocho tipos de asfalto sujetos a un proceso controlado de oxidación. Adicionalmente se presentan resultados de desempeño de la mezcla asfáltica en relación con el daño por humedad en Moisture Induced Sensitivity Test-MIST y su análisis a través de imágenes con el programa iPAS $2 \mathrm{D}^{\circ}$, desarrollado por el grupo MARC, Universidad de Wisconsin -Madison y liderado por el profesor Hussain Bahia. Los resultados indican un mayor porcentaje de pérdida de resistencia a la tracción cuando las mezclas se ensayan en el equipo MIST respecto al ensayo Tensile Strength Ratio-TSR. De igual forma, el análisis con imágenes muestra la evolución de pérdida de contacto de los agregados de acuerdo con los diferentes periodos de oxidación y su relación con la susceptibilidad a la humedad MIST.

PALABRAS CLAVE: Moisture Induced Sensitivity Test-MIST, Tensile Strength Ratio-TSR, iPAS 2D ${ }^{\circ}$ software, ligantes asfálticos sumergidos.

\section{ABSTRACT}

Misture Damage is one of the most common problems in asphalt pavements roads in tropical countries and in areas exposed to prolonged periods of rain. This research presents the results of moisture damage in a dense asphaltic mixture, which was designed by the Superpave ${ }^{\circ}$ methodology and manufactured with eight types of asphalt, subjected to a controlled process of oxidation. In addition, the results of the performance of the asphalt mixture and their relation with moisture damage in Moisture Induced Sensitivity Test-MIST and its analysis using the $i P A S 2 D^{\circ}$ software, which was developed by the MARC Group from the University of Wisconsin-Madison and led by the Professor Hussain Bahia. The results indicate a higher loss of tensile strength when the mixtures are tested with the MIST test compared with the Tensile Strength Ratio-TSR test. Similarly, the images analysis shows the evolution of loss of contact of the aggregates according to the different periods of oxidation of the asphalts and its relation with the MIST analysis.

KEYWORDS: Moisture Induced Sensitivity Test-MIST, Tensile Strength Ratio-TSR, iPAS $2 D^{\circ}$ software, submerged asphalt binders. 


\section{INTRODUCCION}

El estudio de la susceptibilidad al daño por humedad "Moisture Damage" es uno de los más analizados a nivel mundial debido al impacto económico que tiene en los pavimentos.

La mayoría de los países que tienen problemas en sus carreteras por presencia de agua han tomado medidas para mitigarlo, que van desde el control en los procesos constructivos hasta la aplicación de agentes antistripping. Sin embargo, no siempre los resultados han sido favorables y se ha discutido en diversos espacios, cuál debe ser la solución para evitar los daños generados por humedad.

El problema no es fácil de abordar, porque en este modo de daño se involucran diversas variables: agregados, asfalto, mastic, vacios, adhesión y cohesión, que además se pueden clasificar de acuerdo con el momento en que intervienen y se controlan en la mezcla asfáltica, es decir, durante la etapa del diseño, durante la fabricación y colocación y durante la etapa de servicio.

En esta investigación se hace énfasis sobre los ensayos típicos que permiten evaluar el daño por humedad en la metodología Superpave ${ }^{\bullet}$, el ensayo Tensile Strenght Ratio-TSR y recientemente el Moisture Induced Sensitivity Test- MIST.

El ensayo TSR después de diversos estudios como el desarrollado por (Kennedy et al, 1984), está normalizado y muchos países lo han adoptado en sus especificaciones para el control del daño por humedad de las mezclas asfálticas.

En el ensayo MIST, tras tres horas de acondicionamiento en donde se simula la presión, tráfico y temperatura, se determina el daño por stripping. La medición final del esfuerzo de falla por tensión se realiza de forma similar al ensayo TSR, de tal manera que se cuantifica porcentualmente la pérdida de resistencia a la tracción indirecta en condición seca y húmeda.

Los asfaltos utilizados en la investigación fueron sometidos a un proceso controlado de oxidación y se denominan: S0, S3, S6, S9, S12, S15, S18 y S21. Para determinar el daño por humedad se fabricaron ocho tipo de mezclas asfálticas con cada uno de estos asfaltos y se analizaron con el software iPAS $2 \mathrm{D}^{\circ}$ desarrollado por el grupo MARC de la Universidad de Wisconsin -Madison y liderado por el profesor Hussain Bahia. Este análisis muestra el efecto de la oxidación del asfalto en la mezcla y el daño por humedad de ésta en relación con el contacto de las partículas en ella. Finalmente se presenta la relación entre el análisis en MIST, TSR y el análisis a través de imágenes de estas mezclas asfálticas. La investigación quiere llamar la atención sobre la necesidad de utilizar métodos dinámicos en el análisis de la humedad de las mezclas así como el análisis con imágenes ya que resultaría interesante desarrollar pruebas de campo no destructivas para controlar estos problemas.

\section{ANTECEDENTES}

El desempeño de una mezcla asfáltica en caliente (HMA) se ve afectado por la presencia del agua ó vapor de agua al interior de la misma, esto se conoce como daño por humedad; desde los años 30s se ha investigado el fenómeno, encontrando que una de sus manifestaciones es el stripping o más conocido como el desprendimiento del agregado pétreo del ligante asfaltico, (Lottman, 1971).

El stripping se puede definir como la pérdida de adherencia entre la película de asfalto y la superficie del agregado en la mezcla asfáltica, debido a la acción del agua, la cual rompe la unión adhesiva entre ambos y ocasiona pérdida de funcionalidad de la vía, (Figueroa et al, 2013). El agua también puede afectar el cemento asfáltico por interacción química y/o físicamente resultando en una reducción de la cohesión, lo que podría conducir a una severa reducción en la integridad y la resistencia de la mezcla, (Kanitpong y Bahía, 2005).

Los mecanismos involucrados en el fenómeno de stripping se definen como: desplazamiento que ocurre por penetración de agua en la superficie de los agregados a través de una ruptura en la película de asfalto; desprendimiento por suciedad, humedad o incompatibilidad mineralógica en los agregados; emulsificación espontánea la cual ocurre cuando el agua y el asfalto se juntan para formar una emulsión invertida, cuando ocurre esto la emulsión penetra en el sustrato y la unión adhesiva se rompe, (Solaimanian et al, 2003) y (Figueroa et al 2013) y la socavación mecánica, que se produce como consecuencia del aumento de la presión en los poros por la carga de tráfico.

Lottman presentó el protocolo del ensayo TSR en 1978, el cual fue un gran avance para predecir el daño por humedad inducido en la mezcla asfáltica. Posteriormente, en 1978, este protocolo fue modificado por Tunnicliff y estandarizado como AASHTO T283 y llamado ensayo de tracción indirecta en mezclas asfálticas.

En el año 2005 el Departamento del Transporte de Mississippi (MDOT) realizó la investigación Laboratory Accelerated Stripping Simulator for Hot Mixture Asphaltic, dirigida por (Buchanan et al, 2005). El estudio consistió en la evaluación de HMA de gradación gruesa utilizando metodología Superpave ${ }^{\circledR}$, utilizando tres escenarios: sin ningún antistripping, con cal hidratada y con cal hidratada, más un agente antistripping líquido. Los ligantes asfálticos evaluados fueron : PG 67-22 (asfalto puro) 
y PG 76-22 (polímero asfalto modificado). Para la compactación se empleo el compactador giratorio de Superpave ${ }^{\circledR}$, las muestras de HMA se compactaron a una altura de $95 \mathrm{~mm}$ y 7 por ciento de vacíos de aire. Posteriormente, las muestras se acondicionaron en el dispositivo MIST. Al completar el acondicionamiento de las muestras, se determinó la resistencia a la tracción y se compararon con la resistencia a la tracción de las muestras secas determinando la "Resistencia de mezclas de pavimentación bituminosas a Stripping - método de vacío de saturación" (MT-63). Las mediciones de turbidez y el pH del agua también se hallaron antes, durante y después de la prueba para las muestras. Además, las mezclas se ensayaron usando el ensayo de ebullición (MT-59) para comparar los resultados obtenidos a partir del MIST. Una de las conclusiones de la investigación fue "El equipo MIST muestra potencial para medir el stripping de HMA. Los datos obtenidos de la relación de cambio en la turbidez indican claramente que alguna forma de stripping se está produciendo durante la prueba. Una investigación adicional para el MIST se debe realizar para determinar los parámetros de prueba. Sin embargo, antes de seguir investigando se debe continuar con varias modificaciones al MIST para mejorar su funcionamiento y la capacidad de evaluación de stripping".

En esta investigación (Buchanan et al, 2005), indicaron que el equipo MIST fue desarrollado para proporcionar un método racional para la evaluación de la susceptibilidad a la humedad de HMA. (Chen et al, 2008), evaluaron el daño por humedad en mezclas asfáltica en caliente, utilizando el ensayo de desempeño simple (SPT) y el ensayo de tracción indirecta (IDT), las mezclas fueron realizadas con ligante asfálticos (PG 64-22) con y sin aditivo antistripping basado en amina (ASA). Las muestras se acondicionaron por cuatro métodos diferentes: (1) un ciclo de congelación-descongelación (FT). (2) dos ciclos de congelación- descongelación (FT). (3) 500 ciclos de presión de poros con el (MIST). (4) 1000 ciclos de presión de poros con el (MIST).

\section{Objetivo}

Esta investigación aporta resultados del ensayo MIST como una contribución a la medición del stripping en condiciones más cercanas a la realidad y considerando algunos de los más notables modos de daño por humedad.

El análisis de las mezclas con imágenes es una técnica que ha venido cobrando fuerza para analizar la microestructura de la mezcla y capturar el número zonas de contacto, longitud y orientación de las mismas. Las investigaciones al respecto han aplicado tecnologías que van desde el análisis no destructivo de imágenes tomadas con tomografía, (Masad et al, 1999a, 1999b), en donde se trabajó con escalas de grises a partir de la densidad de la fase de estudio. Esta investigación se basa en el análisis con iPAS2, que es una versión mejorada de una primera versión que se llamó Image Processing and Analysis System- iPAS, (Sefidmazgi et al, 2012), realizaron una investigación sobre la microestructura de diferentes mezclas en las que se varió la volumetría, el método de compactación y se correlacionaron con el desempeño por ahuellamiento de las mismas.

\section{MATERIALES Y MÉTODOS}

\section{Metodología}

El diseño de la investigación se realizó a partir de la comparación de una mezclas asfáltica densa en caliente fabricada con asfalto proveniente de refinería 80-100 por metodología Superpave ${ }^{\circledR}$, cuyo PG máximo fue $58^{\circ} \mathrm{C}$ y la temperatura de fatiga $19^{\circ} \mathrm{C}$. El asfalto no se clasificó por temperaturas bajo cero debido a

\section{Tabla 1. Caracterización del asfalto}

\begin{tabular}{|c|c|c|c|c|c|c|}
\hline Ensayo & Unidad & Norma de ensayo & $\begin{array}{l}\text { Número de } \\
\text { muestras }\end{array}$ & Valor promedio & Desviación Estándar & $\begin{array}{l}\text { Coeficiente de } \\
\text { variación (\%) }\end{array}$ \\
\hline Penetración a $25^{\circ} \mathrm{C}$ & $0.1 \mathrm{~mm}$ & $\begin{array}{l}\text { ASTMD5-97 AASHTOT } \\
\text { 49-03 NTL 131/81 }\end{array}$ & 18 & 83,22 & 1,11 & 1,34 \\
\hline Punto de ablandamiento & ${ }^{\circ} \mathrm{C}$ & ASTM D36-95(2000) & 12 & 50,55 & 0,51 & 1,01 \\
\hline Índice de Penetración & - & ASTM D113 & - & 0,26 & - & - \\
\hline $\begin{array}{l}\text { Ductilidad de materiales } \\
\text { asfálticos }\end{array}$ & $\mathrm{cm}$ & AASHTO T51 & 6 & 145 & 6 & 4,46 \\
\hline $\begin{array}{l}\text { Punto de llama (copa abierta de } \\
\text { cleveland) }\end{array}$ & ${ }^{\circ} \mathrm{C}$ & ASTM D92.02b & 3 & 319,14 & 2,8 & 0,87 \\
\hline $\begin{array}{l}\text { Punto de ignición (copa abierta } \\
\text { de cleveland) }\end{array}$ & ${ }^{\circ} \mathrm{C}$ & ASTM D92.02b & 3 & 358,3 & 2,8 & 0,78 \\
\hline Gravedad específica & - & ASTM D70-03 & 3 & 1,007 & 0,002 & 0,22 \\
\hline $\begin{array}{l}\text { Viscosidad rotacional Brookfield } \\
\text { a } 135^{\circ} \mathrm{C}\end{array}$ & Pa.s & AASHTO T316-04 & 33 & 0,35 & - & - \\
\hline
\end{tabular}


que este no es un problema en los países tropicales. La mezcla fabricada con los asfaltos: S0, S3, S6, S9, S12, S15, S18 y S21. Para el análisis del daño por humedad se realizaron los ensayos de TSR y MIST. El módulo dinámico se midió en Materials Testing System- MTS según norma de ensayo, INVE 754-2007.

\section{Resultados y Análisis}

El asfalto utilizado es proveniente de refinería cuya clasificación por penetración es de 80-100 (1/10 mm) y la evaluación de sus propiedades se presenta en la Tabla 1.

Los agregados pétreos utilizados en la investigación son de origen metamórfico aluvial y su caracterización se presenta en la Tabla 2.

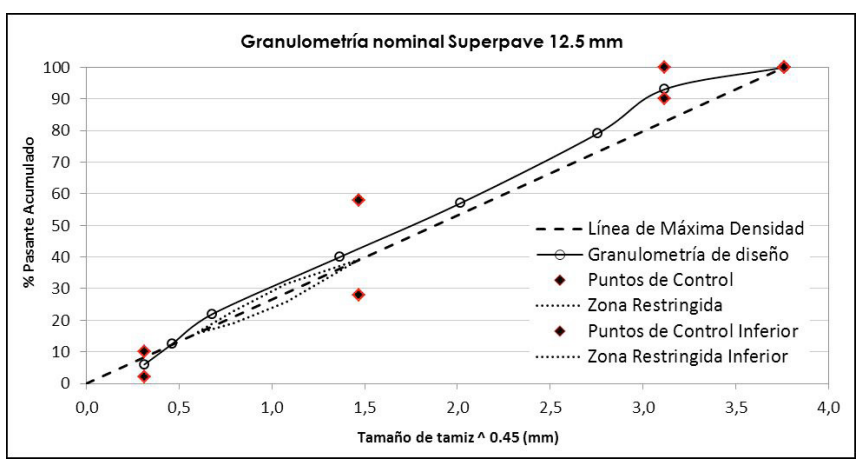

Figura 1. Granulometría de la mezcla

Tabla 2. Caracterización de agregados pétreos

\begin{tabular}{|c|c|c|c|c|c|c|c|c|c|}
\hline Ensayo & Unidad & $\begin{array}{c}\text { Normas } \\
\text { Internacionales de } \\
\text { Referencia }\end{array}$ & $\begin{array}{l}\text { Límite } \\
\text { mínimo }\end{array}$ & $\begin{array}{l}\text { Límite } \\
\text { máximo }\end{array}$ & $\begin{array}{l}\text { Valor } \\
\text { medio } \\
\text { grava } \\
\text { triturada }\end{array}$ & $\begin{array}{l}\text { Valor } \\
\text { medio } \\
\text { material } \\
\text { Fino }\end{array}$ & $\begin{array}{l}\text { Número de } \\
\text { Muestras }\end{array}$ & $\begin{array}{l}\text { Desviación } \\
\text { Estándar }\end{array}$ & $\begin{array}{l}\text { Coeficiente } \\
\text { de } \\
\text { Variación }\end{array}$ \\
\hline $\begin{array}{l}\text { Resistencia al Desgaste de Agregados } \\
<37.5 \mathrm{~mm} \text { por medio de la Máquina de } \\
\text { Los Ángeles }\end{array}$ & $\%$ & $\begin{array}{l}\text { ASTM C } 131-01 \\
\text { AASHTO T } 96-02 \\
\text { UNE EN } 1097-2: 1998\end{array}$ & & $35 \%$ & $28.59 \%$ & $\mathrm{~N} / \mathrm{A}$ & 3 & $0.62 \%$ & 0.02 \\
\hline $\begin{array}{l}\text { Desgaste por Abrasión utilizando el } \\
\text { Aparato MicroDeval }\end{array}$ & $\%$ & $\begin{array}{l}\text { AASHTO T327 - } 05 \\
\text { ASTM D } 6928-03 \\
\text { UNE EN } 1097-1\end{array}$ & & $20 \%$ & $10.38 \%$ & $\mathrm{~N} / \mathrm{A}$ & 3 & $0.26 \%$ & 0.02 \\
\hline Ensayo de solidez agregados gruesos & \multirow{2}{*}{$\%$} & \multirow{2}{*}{$\begin{array}{l}\text { DNER-ME } 096-98 \\
\text { BS } 812 \text { Part } 110-1990\end{array}$} & & \multirow{2}{*}{$18 \%$} & $1.10 \%$ & - & 3 & $0.15 \%$ & 0.25 \\
\hline Ensayo de solidez de agregados finos & & & & & - & $4.10 \%$ & 4 & $0.28 \%$ & 0.07 \\
\hline $\begin{array}{l}\text { Valor de Azul de Metileno en Agregados } \\
\text { Finos y Llenantes Minerales }\end{array}$ & $\mathrm{mg} / \mathrm{g}$ & AASHTO TP 57-01 (200 & - & 10 & N/A & 4.83 & 3 & 0.14 & 0.03 \\
\hline Índice de Alargamiento (IL) & \multirow{3}{*}{$\%$} & \multirow{3}{*}{ INV 230} & & & $6.92 \%$ & N/A & 3 & $0.99 \%$ & 0.14 \\
\hline Índice de Aplanamiento (IA) & & & & & $21.09 \%$ & $\mathrm{~N} / \mathrm{A}$ & 3 & $0.69 \%$ & 0.03 \\
\hline $\begin{array}{l}\text { Índice de alargamiento y aplanamiento } \\
\text { (IAL) }\end{array}$ & & & & & $2.54 \%$ & N/A & 3 & $0.46 \%$ & 0.18 \\
\hline $\begin{array}{l}\text { Porcentaje de Caras Fracturadas en los } \\
\text { Agregados }\end{array}$ & $\%$ & ASTM D 5821 - 01 & $75 \%$ & - & $87.68 \%$ & N/A & 3 & $2.42 \%$ & 0.03 \\
\hline $\begin{array}{l}\text { Partículas Planas y/o Alargadas en } \\
\text { Agregados Gruesos }\end{array}$ & & \multirow{3}{*}{ ASTM D 4791 - 99} & \multirow{3}{*}{-} & \multirow{3}{*}{$10 \%$} & - & - & - & - & - \\
\hline Partículas aplanadas & \multirow{2}{*}{$\%$} & & & & $0.00 \%$ & - & 3 & $0.00 \%$ & - \\
\hline Partículas aplanadas y alargadas & & & & & $0.10 \%$ & - & 3 & $0.13 \%$ & 1.33 \\
\hline $\begin{array}{l}\text { Equivalente de Arena Suelos y Agregados } \\
\text { finos }\end{array}$ & $\%$ & $\begin{array}{l}\text { ASTM D } 2419-95 \\
\text { AASHTOT } 176-02\end{array}$ & $50 \%$ & & - & $57.33 \%$ & 3 & $5.13 \%$ & 0.09 \\
\hline Gravedad Específica Bulk (gravas) & & \multirow{4}{*}{$\begin{array}{l}\text { ASTM C } 127-88 \\
\text { (Reaprobada en el 2001) }\end{array}$} & \multirow{4}{*}{\multicolumn{2}{|c|}{ según diseño }} & 2.59 & - & 3 & 0.02 & 0.01 \\
\hline Gravedad Específica Bulk sss (gravas) & & & & & 2.63 & - & 3 & 0.02 & 0.01 \\
\hline Gravedad Específica Bulk aparente (gravas) & & & & & 2.70 & - & 3 & 0.03 & 0.01 \\
\hline Absorción de Agregados (gravas) & $\%$ & & & & $1.63 \%$ & - & 3 & $0.80 \%$ & 0.49 \\
\hline Gravedad Específica Bulk (arena) & & \multirow{4}{*}{$\begin{array}{l}\text { ASTM C } 127-88 \\
\text { (Reaprobada en el 2001) }\end{array}$} & \multirow{4}{*}{\multicolumn{2}{|c|}{ según diseño }} & - & 2.48 & 3 & 0.01 & 0.00 \\
\hline Gravedad Específica Bulk sss (arena) & & & & & - & 2.52 & 3 & 0.01 & 0.00 \\
\hline Gravedad Específica Bulk aparente (arena) & & & & & - & 2.59 & 3 & 0.01 & 0.00 \\
\hline Absorción de Agregados (arena) & $\%$ & & & & - & $1.73 \%$ & 3 & $9.48 \%$ & 5.48 \\
\hline Gravedad Específica Bulk (llenante) & & $\begin{array}{l}\text { ASTM C } 128-97 \\
\text { AASHTO T } 84-00 \\
(2004) \\
\text { NLT } 154-92\end{array}$ & según & diseño & - & 2.73 & 3 & 0.00 & 0.00 \\
\hline
\end{tabular}


Tabla 3. Volumetría de la mezcla de diseño

\begin{tabular}{|c|c|c|c|}
\hline \multicolumn{4}{|c|}{ Volumetría de diseño mezcla MDC-2 } \\
\hline $\begin{array}{c}\text { Contenido de } \\
\text { asfalto en \% }\end{array}$ & $\begin{array}{c}\text { vacíos de aire } \\
\text { en \% }\end{array}$ & \begin{tabular}{c} 
vacíos en el agregado \\
mineral en \% \\
\cline { 3 - 4 }
\end{tabular} & $\begin{array}{c}\text { vacíos llenos de } \\
\text { asfalto en \% }\end{array}$ \\
\hline 5.7 & 4.1 & 14.2 & $(\%$ VFA) \\
\hline
\end{tabular}

La mezcla asfáltica se diseñó por metodología Superpave ${ }^{\bullet}$ y la granulometría seleccionada se encuentra marcada por la franja resaltada en el porcentaje retenido, Figura 1.

La medición del módulo dinámico se realizó con la MTS que es una máquina de ensayo electro- hidráulica con un generador que puede producir una onda de medio seno inverso, además tiene la capacidad de aplicar las cargas dentro de un intervalo de frecuencias que varían de 0.1 hasta $20 \mathrm{~Hz}$ y niveles de esfuerzo hasta $690 \mathrm{kPa}\left(100 \mathrm{lbs} / \mathrm{pulg}^{2}\right)$. El ensayo se realizó de acuerdo con la norma de la American Society for Testing and MaterialsASTM D3497-97. Para determinar el módulo dinámico, se aplicaron cargas sinusoidales a la muestra de prueba a diferentes temperaturas y diferentes frecuencias según la norma. El módulo dinámico se determinó como la relación entre el esfuerzo aplicado y la respuesta resultante recuperable de la deformación axial de la muestra. La curva maestra de la mezcla fabricada con el asfalto original se presenta a continuación. Ver Figura 2.

\section{Ensayo TSR vs. Ensayo MIST}

La evaluación de la susceptibilidad a la humedad en la metodología Superpave ${ }^{\oplus}$, se realiza con el ensayo TSR, en esta investigación además de este ensayo se realizó el MIST, ver Figura 3.

Este ensayo establece el procedimiento para medir el efecto del agua en las mezclas asfálticas al realizar la medición del esfuerzo de tensión indirecta. En el ensayo de Lottman modificado, el vacío de saturación es del $70 \%$ y $80 \%$, la temperatura y tasa de carga durante el ensayo son de $25^{\circ} \mathrm{C}$ y $5,08 \mathrm{~cm} / \mathrm{min}$, respectivamente. La susceptibilidad a la humedad se determina preparando un set de especímenes compactados en laboratorio con un rango de vacíos entre el 6\% y el $8 \%$. Este grupo se subdivide en dos grupos que tengan el mismo contenido de vacíos cada uno, con el propósito de compararlos en condición seca y húmeda. El grupo que se humedece tiene el acondicionamiento descrito anteriormente. El daño por humedad se determina por la relación entre el esfuerzo de tensión del subgrupo en condición húmeda respecto al esfuerzo de tensión del subgrupo en condición seca.

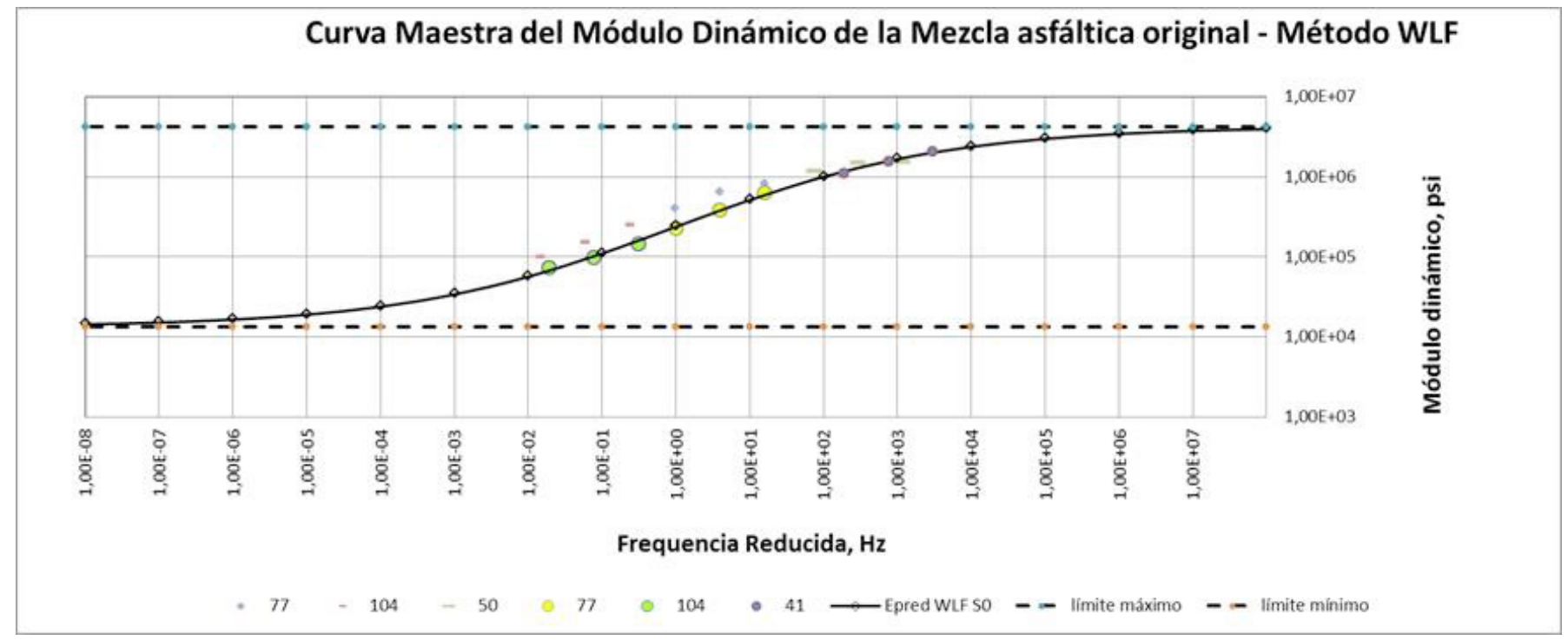

Figura 2. Curva maestra del módulo dinámico de la mezcla asfáltica

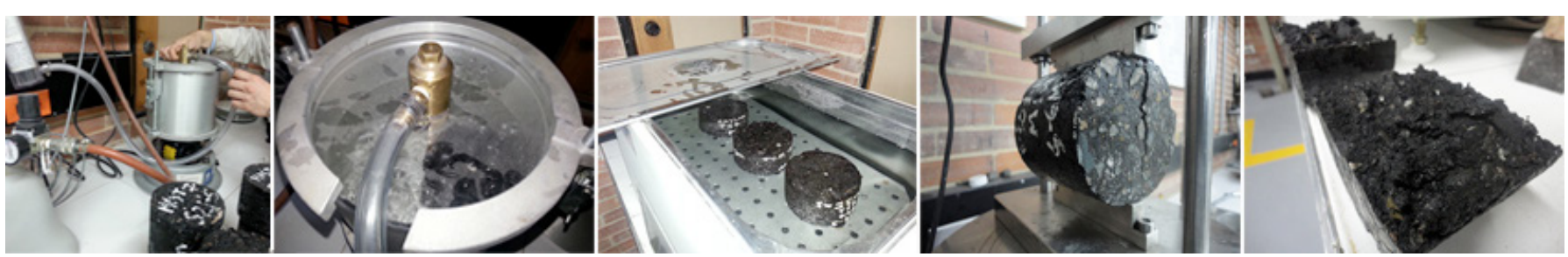

Figura 3. Ensayo TSR para determinar la susceptibilidad a la humedad 
Algunas de las especificaciones y documentos que definen el ensayo son la AASHTO T283, NCHRP Report 274, (Tunnicliff y Root, 1984) y Tex 531-C.

El ensayo MIST es un ensayo de acondicionamiento acelerado, ver Figura 4, para determinar la susceptibilidad a la humedad de la mezcla asfáltica y por lo tanto la posibilidad de stripping en la misma. El ensayo se realiza a temperaturas mayores a la temperatura ambiente y crea una presión de poros dentro de la mezcla compactada para lograr los efectos que esta experimenta bajo cargas vehiculares. El ensayo reproduce tres factores: esfuerzo, presión y alta temperatura en presencia de agua, (Xinjun Li et al, 2010). Una vez terminado el acondicionamiento se mide el esfuerzo de tensión indirecta tal como se hace en el ensayo TSR. El daño por humedad se determina por la relación entre el esfuerzo de tensión en la condición húmeda respecto al esfuerzo de tensión en la condición seca.

De acuerdo con los ensayos TSR y MIST se observa que para este último ensayo la pérdida de contactos fue mayor, notándose una mayor degradación de la mezcla asfáltica. Ver Tabla 4.

Durante la investigación se trabajó con los asfaltos de S0 a S21 con los cuales se realizó el ensayo TSR y MIST con el propósito de observar la relación entre los dos ensayos y los resultados obtenidos. Para todos los asfaltos la susceptibilidad a la humedad fue mayor para el ensayo MIST, ver Figura 5. El acondicionamiento con el ensayo MIST es más aproximado a la realidad de la mezcla asfáltica en servicio. Durante el ensayo es deseable que se aplique una temperatura $\mathrm{T}=60^{\circ} \mathrm{C}$, que corresponde a la máxima de la mezcla en servicio un número de ciclos según el tráfico, liviano, mediano o pesado, para esta investigación se aplicaron 3000 ciclos (tráfico alto) y una presión de 50 psi.

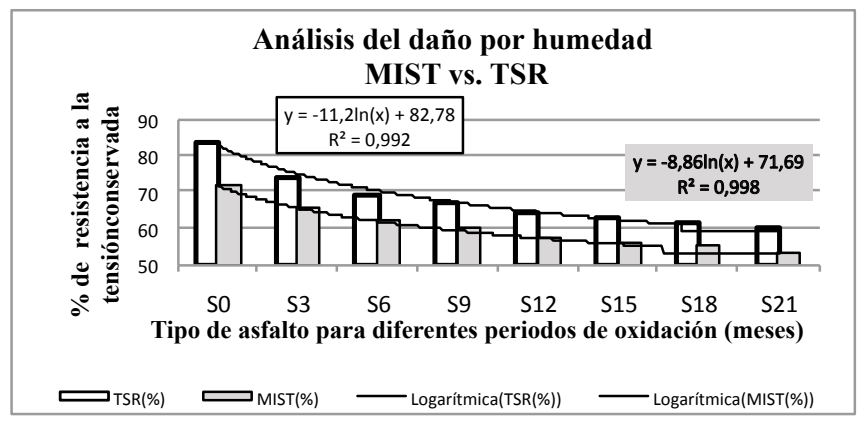

Figura 5. Gráfica comparativa entre el ensayo TSR y el MIST

\section{Análisis de Imágenes a Través del Programa iPAS 2D ${ }^{\circledR}$}

El procesamiento y análisis de imágenes se realizó a través del programa iPAS ${ }^{\odot} 2 \mathrm{D}$ creado por el grupo MARC de la Universidad de Wisconsin-Madison. Para el presente estudio se determinó tomar las probetas del ensayo TSR y MIST con un porcentaje de vacíos de aire de $7 \%$ para los distintos periodos de inmersión.
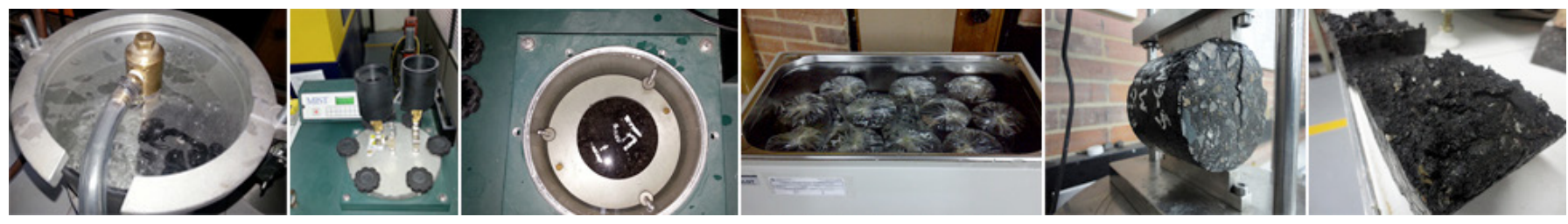

Figura 4. Ensayo MIST para determinar la susceptibilidad a la humedad

Tabla 4. Variación del ensayo TSR y MIST en relación al número de contactos analizados con iPAS 2D ${ }^{\circledR}$

\begin{tabular}{|c|c|c|c|c|}
\hline Tipo de asfalto & Ensayo TSR (\%) & Ensayo MIST (\%) & Relación N ${ }^{\circ}$ de contactos TSR (\%) & ${\text { Relación N }{ }^{\circ} \text { de contactos MIST (\%) }}^{8}$ \\
\hline S0 (sin sumergir) & 83.85 & 71.92 & 94.77 & 88.52 \\
\hline S3 (sumergido 3 meses) & 74.23 & 65.42 & 91.34 & 86.68 \\
\hline S6 (sumergido 6 meses) & 69.51 & 61.62 & 88.73 & 84.32 \\
\hline S9 (sumergido 9 meses) & 66.86 & 59.49 & 85.41 & 78.19 \\
\hline S12 (sumergido 12 meses) & 64.40 & 57.25 & 82.86 & 75.56 \\
\hline S15 (sumergido 15 meses) & 62.96 & 55.89 & 80.21 & 72.60 \\
\hline S18 (sumergido 18 meses) & 61.26 & 54.87 & 78.38 & 69.35 \\
\hline S21 (sumergido 21 meses) & 60.03 & 53.13 & 76.68 & \\
\hline
\end{tabular}



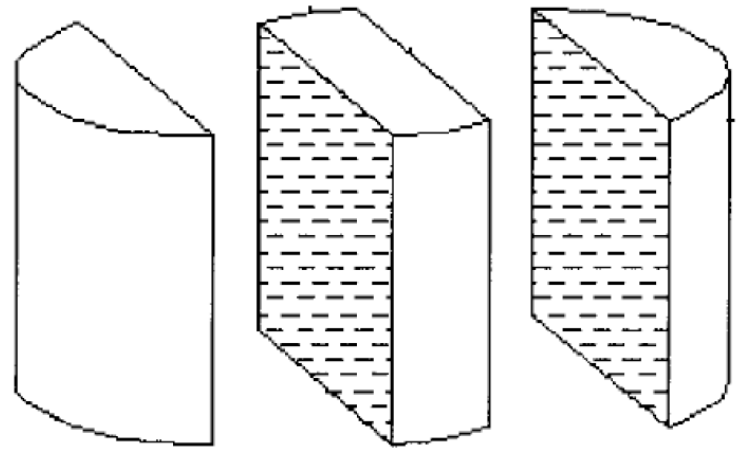

Figura 6. Seccionamiento patrón de las muestras de mezcla asfáltica

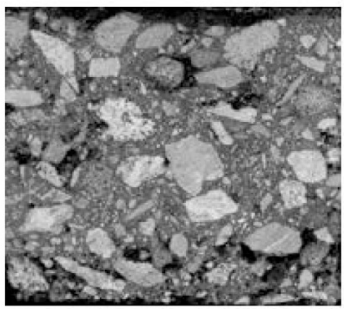

Imagen Original

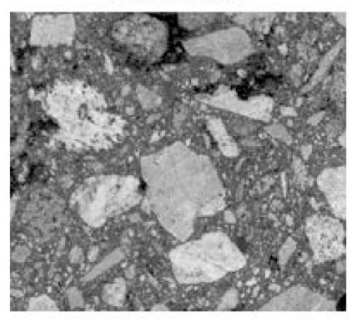

Filtro Medio

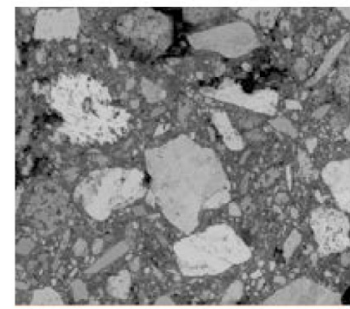

Filtro Máximo

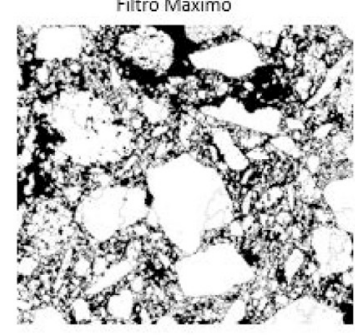

Imagen de blanco y negro

Cada una de ellas se cortó en dos secciones tanto para el estado seco y saturado, como se muestra en la Figura 6, con el propósito de determinar la cantidad y área de contactos entre agregados en relación con los periodos de inmersión del asfalto.

Cada una de las secciones se escaneó con una resolución de 1200 puntos por pulgada (ppp) en escala de grises, la cual en la etapa final del procesamiento se convirtió en una imagen de blanco y negro que se utilizó para el análisis de la microestructura; sin embargo, hay varios pasos para mejorar esta imagen de las diferentes fases llamados filtros, ver Figura 7, el software emplea un filtro medio, el máximo filtro y el umbral que indica el límite de nivel de escala de grises que separa el asfalto de los agregados los cuales son parámetros de entrada (datos variables).

La aplicación de filtros se detiene hasta que el área de fracción de los agregados de la imagen escaneada versus la fracción de volumen calculado tengan el mismo valor, como se puede observar en la Figura 8 la cual muestra la curva de degradación en el laboratorio vs la escaneada.

Los datos de entrada complementarios son las propiedades de la muestra como: vacíos totales de la mezcla, porcentaje de asfalto, gravedad específica de los agregados y del ligante, curva de gradación obtenida en el laboratorio (datos invariables) y específicamente para el análisis, se ingresa la distancia de separación mínima entre agregados, para ser considerada un contacto y el mínimo tamaño de agregado para buscar como contacto, estos dos parámetros fueron fijos durante el procesamiento realizado. En el análisis de imágenes el software permite encontrar el número y longitud de contactos y su orientación. Ver Figura 9.

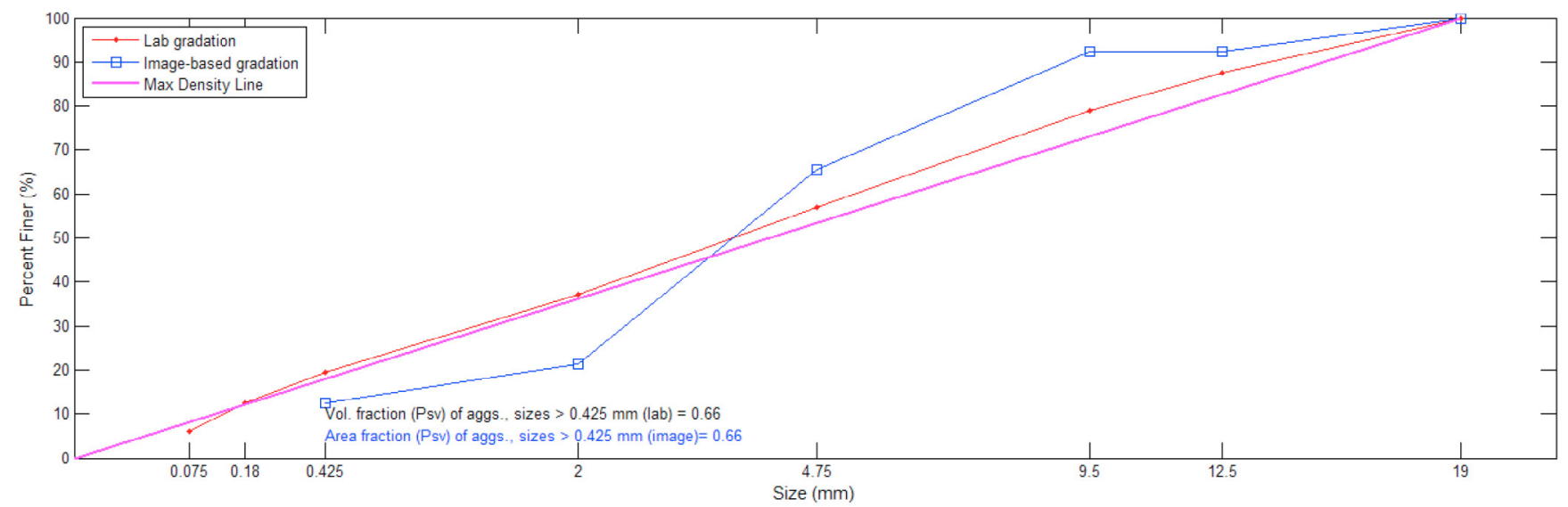

Figura 8. Control de la imagen en el programa iPAS 2D ${ }^{\odot}$ 


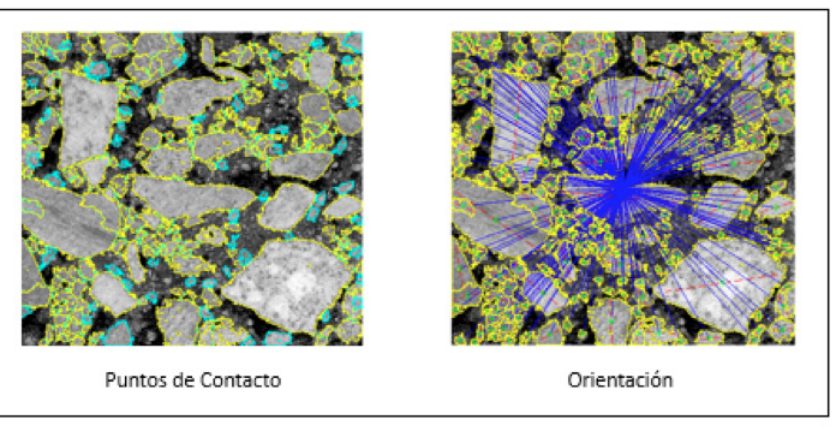

Figura 9. Análisis de imágenes en el programa iPAS 2D ${ }^{\oplus}$

Después de procesar las imágenes en el software iPAS $2 \mathrm{D}^{\circ} \mathrm{y}$ encontrar los puntos de contacto de la estructura interna de la muestra analizada, el software permite determinar cuántos contactos entre agregado-agregado se encuentran en la muestra, determinando el número de agregados analizados. Ver Figura 10.

\section{DISCUSIÓN Y CONCLUSIONES}

El acondicionamiento con el ensayo MIST es más aproximado a la realidad de la de la mezcla asfáltica en servicio. Durante el ensayo es deseable que se aplique una temperatura $\mathrm{T}=60^{\circ} \mathrm{C}$, que corresponde a la máxima de la mezcla en servicio, un número de ciclos según el tráfico, liviano, mediano o pesado, para esta investigación se aplicaron 3000 ciclos y una presión de 50 psi.

El acondicionamiento previo al ensayo TSR solo considera el control de la temperatura en una condición estática, sin presión ni ciclos de carga, así mismo, no es posible observar el daño por stripping que tiene la mezcla. Si bien el ensayo indica una pérdida de resistencia por efecto del agua que no necesariamente está relacionada con las condiciones de campo. Es necesario realizar trabajos complementarios sobre la relación entre estos dos ensayos y calibrar los resultados del MIST con pruebas de campo que permitan establecer una correlación y confiabilidad de la prueba.

Vale la pena tener en cuenta que el ensayo MIST desde el momento del acondicionamiento, con las briquetas compactadas hasta el momento de finalización del mismo, toma aproximadamente cuatro horas. Este dato es importante para considerar el plan de ensayos del proyecto.

El acondicionamiento previo al ensayo TSR solo considera el control de la temperatura en una condición estática, sin presión ni ciclos de carga, así mismo no es posible observar el daño por stripping que tiene la mezcla. Si bien el ensayo indica una pérdida de resistencia por efecto del agua no necesariamente está relacionada con las condiciones de campo.

Después de procesar las imágenes en el software iPAS $2 \mathrm{D}^{\circledR} \mathrm{y}$ encontrar los puntos de contacto de la estructura interna de la muestra analizada, el software permite determinar cuántos contactos entre agregado-agregado se encuentran en la muestra, determinando el número de agregados analizados. Para este caso la mayor pérdida de contacto se observó para las muestras ensayadas en MIST y en la condición húmeda.

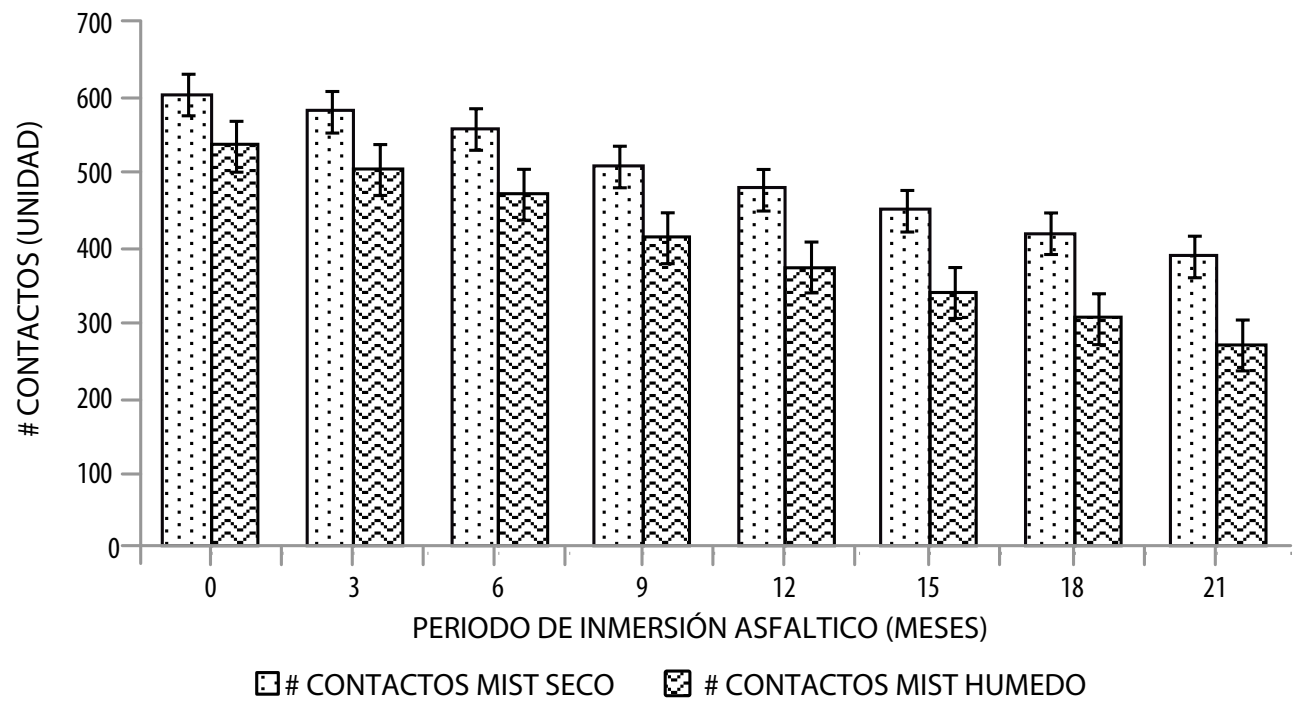

Figura 10. Consolidado \# de contactos MIST 7\% 


\section{REFERENCIAS BIBLIOGRÁFICAS}

1. $\quad$ ASTM, A., Test, W. A., Test, C. S., \& Test, A. R. (1950). American Society for Testing and Materials (ASTM).

2. American Association of State Highway and Transportation Officials-AASHTO Standards

3. Buchanan, M. S., \& Moore, V. M. (2005). Laboratory accelerated stripping simulator for hot mix asphalt (No. FHWA/MS-DOT-RD-04-167,).

4. Chen, X., \& Huang, B. (2008). Evaluation of moisture damage in hot mix asphalt using simple performance and superpave indirect tensile tests. Construction and Building Materials, 22(9), 1950-1962.

5. Cubillos Carlos \& Nuñez Camila. (2014). Análisis de la susceptibilidad al daño por humedad de una mezcla asfáltica para rodadura empleando asfaltos sumergidos. Pontificia Universidad Javeriana.

6. Figueroa, A., Velasquez, R., Reyes, F., \& Bahia, H. (2013). Effect of Water Conditioning for Extended Periods on the Properties of Asphalt Binders. Transportation Research Record: Journal of the Transportation Research Board, (2372), 34-45.

7. Instituto Nacional de Vías -INVIAS -Normas. (2007). Colombia.

8. Kanitpong, K., \& Bahia, H. (2005). Relating adhesion and cohesion of asphalts to the effect of moisture on laboratory performance of asphalt mixtures. Transportation Research Record: Journal of the Transportation Research Board, (1901), 33-43.

9. Kennedy, T. W.; Roberts, F. L.; and Anagnos, J. N. (1984). Research Report 253-5, Center for Transportation Research, University of Texas at Austin.

10. Lottman, R. P. (1971). The moisture mechanism that causes asphalt stripping in asphaltic pavement mixtures.

11. Masad, E., Muhunthan, B., Shashidhar, N., \& Harman, T. (1999a). Internal structure characterization of asphalt concrete using image analysis. Journal of computing in civil engineering, 13(2), 88-95.

12. Masad, E., Muhunthan, B., Shashidhar, N., \& Harman, T. (1999b). Quantifying laboratory compaction effects on the internal structure of asphalt concrete. Transportation Research Record: Journal of the Transportation Research Board, (1681), 179-185.

13. Sefidmazgi, N. R., Tashman, L., \& Bahia, H. (2012). Internal structure characterization of asphalt mixtures for rutting performance using imaging analysis. Road materials and pavement design, 13(sup1), 21-37.

14. Solaimanian, M., Harvey, J., Tahmoressi, M., \& Tandon, V. (2003, February). Test methods to predict moisture sensitivity of hot-mix asphalt pavements. In Transportation Research Board National Seminar. San Diego, California (pp. 77-110).

15. Tunnicliff, D. G.; and Root, R. E. (1984). “Use of Anti-Stripping Additives in Asphaltic Concrete Mixtures". NCHRP Report 274, Laboratory Phase, TRB, National Research Council, Washington, D.C.

16. Xinjun Li, Nelson Gibson, Evaluation Moisture Induced Sensitivity Test (MIST) Device: Comparison and Contrast with AASHTO T 283. (2010). FHWA, Draft Report. 